\title{
FE-SEM Studies of a Hypermineralized Bone Material
}

\author{
Z. Li and J.D. Pasteris \\ Department of Earth and Planetary Sciences, Washington University in St. Louis, St. Louis, MO \\ 63130
}

Bone has three main components: mineral (carbonated hydroxylapatite), organic material (mainly type I collagen), and water [1]. Mineral is the major component in bone, e.g., 55 wt.\% in the human femur [2]. The dilemma for the researcher of bone mineral is that abundant collagen masks the real mineral structure, whereas processes of deproteinization, e.g., bleaching and ultrasonic cleaning, may alter the structure and chemistry of the mineral. A hypermineralized bone material would be ideal. Thus, we chose to study the central core region of the rostrum of the male whale $M$. densirostris, which has $\sim 96 \mathrm{wt} . \%$ mineral [2].

FE-SEM (Field emission scanning electron microscopy) has been widely used to study apatite in bone materials [3]. The simple preparation of the samples ensures that the original structure, composition, and morphology are preserved. One $\sim 1$-millimeter particle fractured from the hypermineralized core region of the rostrum was coated by gold for FE-SEM imaging.

Typical areas of the central rostrum are composed of dense mineral prisms, as seen in Fig. 1A. In contrast to images of bones of all other vertebrates, no collagen fibrils were revealed among the mineral prisms by FE-SEM, presumably due to their rarity. The prisms have irregular shapes with lengths of 1-2 $\mu \mathrm{m}$ and widths of several hundred nanometers (Fig. 1A). The rough surface of the prisms, formed by organized mineral bundles, can be distinguished from other bone materials at similar magnification (with no such prismatic features at the micrometer scale), as we observed and as reported by several groups [4,5]. Fig. 1B shows an osteocytic lacuna with a diameter of $\sim 10 \mu \mathrm{m}$ exposed on a fractured surface. The size is very close to that of lacunae in, for instance, rabbit and bovine femora [5], but the inside surface of the rostral lacuna has no texture indicative of collagen fibers. The imaged transitional area between the typical densely packed and a more open mineral morphology in the rostrum (Fig. 1C) may show the onset of new mineralization. The open structure is analogous to clusters of freshly precipitated, synthetic carbonated apatite [6]. The platelets in the open structure have thicknesses of $\sim 40$ nanometers (see Fig. 1D), which is larger than the reported value ( 2 nanometers) for single crystals in bioapatite [4]. Hence, the platelets in Fig. 1D could be stacks of several single plate-like nanocrystals.

In summary, the central core rostrum of the adult male $M$. densirostris is an extremely highly mineralized, well organized bone material. The material therefore is very promising for future investigation of the detailed structure and chemistry of bone mineral. 
[1] M.J. Glimcher et al., Rev. Mineral Geochem, 64 (2006) 223.

[2] K.D. Rogers et al., J. Mater. Sci. L. 18 (1999) 651.

[3] F.H. Jones Surf. Sci. Rep. 42 (2001) 79.

[4] T. Hassenkam et al., Bone 35 (2004) 4.

[5] U.E. Pazzaglia et al., Clin. Orthop. Relat. Res. 467 (2009) 2446.

[6] G. He et al., Nat. Mater. 2 (2003) 552.

[7] This research was partially funded by NIH grant 1R21AR055184-01A2 .
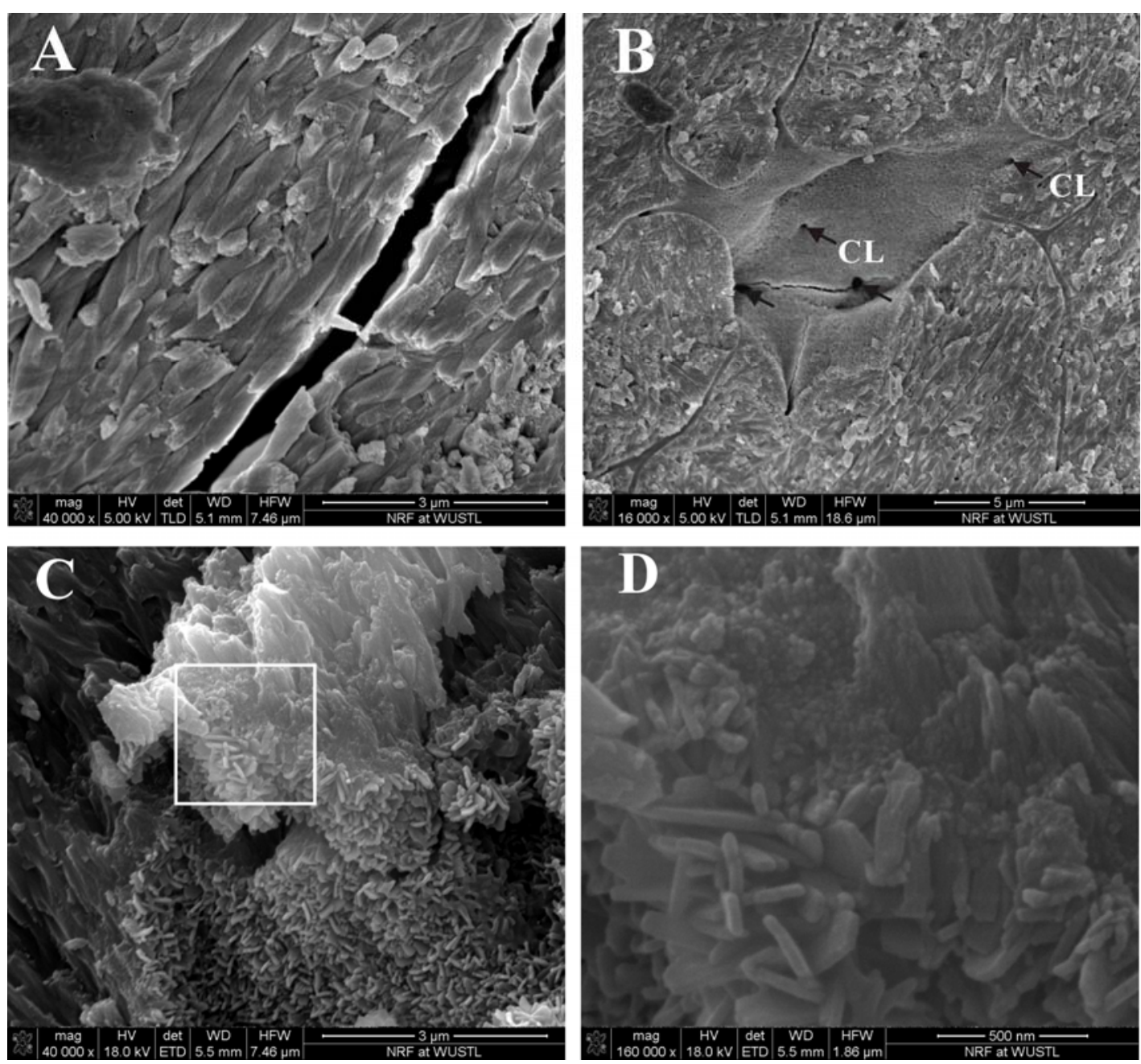

FIG. 1. FE-SEM images of longitudinally oriented rostrum: A: Elongated mineral prisms in a typical area are seen along a crack. B: One lacuna on the fractured surface with its connecting canaliculus system (CL). Openings of canaliculi can be seen inside of the lacuna (arrows). C: A transitional area between densely packed and open mineral structure D: The open structure (enlargement of the square in Fig. 1C) shows platelets with a thickness of $\sim 40$ nanometers. 